\title{
Predictors of cognitive impairment in primary age-related tauopathy: an autopsy study
}

Megan A. lida ${ }^{1 \dagger}$, Kurt Farrell ${ }^{1 \dagger}$, Jamie M. Walker ${ }^{2}$, Timothy E. Richardson², Gabriel A. Marx', Clare H. Bryce ${ }^{1}$, Dushyant Purohit ${ }^{1}$, Gai Ayalon ${ }^{3}$, Thomas G. Beach", Eileen H. Bigio5, Etty P. Cortes ${ }^{1}$, Marla Gearing ${ }^{6}$, Vahram Haroutunian ${ }^{7,8}$, Corey T. McMillan ${ }^{9}$, Edward B. Lee ${ }^{20}$, Dennis W. Dickson ${ }^{10}$, Ann C. McKee ${ }^{11}$, Thor D. Stein ${ }^{11}$, John Q. Trojanowski ${ }^{12}$, Randall L. Woltjer ${ }^{13}$, Gabor G. Kovacs ${ }^{14,15,16}$, Julia K. Kofler ${ }^{17}$, Jeffrey Kaye ${ }^{18}$, Charles L. White $\mathrm{II}^{19}$ and John F. Crary ${ }^{1 *}$ (D)

\begin{abstract}
Primary age-related tauopathy (PART) is a form of Alzheimer-type neurofibrillary degeneration occurring in the absence of amyloid-beta (A $\beta$ ) plaques. While PART shares some features with Alzheimer disease (AD), such as progressive accumulation of neurofibrillary tangle pathology in the medial temporal lobe and other brain regions, it does not progress extensively to neocortical regions. Given this restricted pathoanatomical pattern and variable symptomatology, there is a need to reexamine and improve upon how PART is neuropathologically assessed and staged. We performed a retrospective autopsy study in a collection $(n=174)$ of post-mortem PART brains and used logistic regression to determine the extent to which a set of clinical and neuropathological features predict cognitive impairment. We compared Braak staging, which focuses on hierarchical neuroanatomical progression of AD tau and AB pathology, with quantitative assessments of neurofibrillary burden using computer-derived positive pixel counts on digitized whole slide images of sections stained immunohistochemically with antibodies targeting abnormal hyperphosphorylated tau ( $p$-tau) in the entorhinal region and hippocampus. We also assessed other factors affecting cognition, including aging-related tau astrogliopathy (ARTAG) and atrophy. We found no association between Braak stage and cognitive impairment when controlling for age $(p=0.76)$. In contrast, $\mathrm{p}$-tau burden was significantly correlated with cognitive impairment even when adjusting for age $(p=0.03)$. The strongest correlate of cognitive impairment was cerebrovascular disease, a well-known risk factor $(p<0.0001)$, but other features including ARTAG $(p=0.03)$ and hippocampal atrophy $(p=0.04)$ were also associated. In contrast, sex, APOE, psychiatric illness, education, argyrophilic grains, and incidental Lewy bodies were not. These findings support the hypothesis that comorbid pathologies contribute to cognitive impairment in subjects with PART. Quantitative approaches beyond Braak staging are critical for advancing our understanding of the extent to which age-related tauopathy changes impact cognitive function.
\end{abstract}

Keywords: PART, Dementia, Aging, Braak, ARTAG

*Correspondence: john.crary@mountsinai.org

†Megan A. lida and Kurt Farrell contributed equally to this work

${ }^{1}$ Department of Pathology, Nash Family Department of Neuroscience,

Ronald M. Loeb Center for Alzheimer's Disease, Icahn School of Medicine

At Mount Sinai, Friedman Brain Institute, Neuropathology Brain Bank \&

Research CoRE, 1 Gustave L. Levy Place Box 1194, New York, NY 10029,

USA

Full list of author information is available at the end of the article

\section{Introduction}

It is widely recognized that abnormal hyperphosphorylated tau (p-tau) deposition is a ubiquitous feature of the aging human brain, observed in both cognitively normal subjects and in those with a range of clinical features, including cognitive, motor and psychiatric symptoms original author(s) and the source, provide a link to the Creative Commons licence, and indicate if changes were made. The images or other third party material in this article are included in the article's Creative Commons licence, unless indicated otherwise in a credit line to the material. If material is not included in the article's Creative Commons licence and your intended use is not permitted by statutory regulation or exceeds the permitted use, you will need to obtain permission directly from the copyright holder. To view a copy of this licence, visit http://creativecommons.org/licenses/by/4.0/. The Creative Commons Public Domain Dedication waiver (http://creativeco mmons.org/publicdomain/zero/1.0/) applies to the data made available in this article, unless otherwise stated in a credit line to the data. 
[35]. The causes of tauopathy are diverse, and include both genetic and environmental risk factors [46]. Autosomal dominant mutations in the tau gene (MAPT) cause frontotemporal lobar degeneration and common risk alleles, notably the MAPT 17q21.31 H1 haplotype, are associated with sporadic tauopathies including progressive supranuclear palsy (PSP), corticobasal degeneration (CBD), and argyrophilic grain disease (AGD) [10]. Abnormal p-tau deposition is also seen following exposure to repetitive head trauma in contact sports and other contexts in the setting of chronic traumatic encephalopathy (CTE) [41]. Neurofibrillary tangles (NFT) are also a component of Alzheimer disease (AD), where they are associated amyloid-beta deposits [14].

Although it is generally understood that autopsy studies are critical for establishing definitive diagnoses, the neuropathology of the tauopathies is complex and overlapping. Further, non-impaired individuals often display significant amounts of p-tau accumulation, complicating our understanding of the contribution of such brain changes to symptomatology. Approaches to assessing tauopathy in post-mortem tissues continue to evolve. Neuropathologically, tauopathies can be differentiated by the neuroanatomical regionality of p-tau aggregates, cell type involvement (i.e., neurons versus glia), preferential isoform accumulation, and filament ultrastructure. Based upon these differentiating features, validated neuropathological diagnostic consensus criteria have been devised and, in some cases, undergone revision. Examples include revision of the AD diagnostic criteria, and consensus criteria for CTE $[39,44]$. The term aging-related tau astrogliopathy (ARTAG), which was described in recent consensus criteria on various patterns of astrocytic p-tau observed in aging, has been especially helpful for differentiating age-related changes from CTE, both of which have perivascular p-tau deposits, but with differences in cell types involved [36, 40]. The introduction of criteria for primary age-related tauopathy (PART) to describe individuals who develop AD-type neurofibrillary pathology with or without dementia in the absence of significant amyloid deposition helped to better define this entity and differentiated it from AD [15]. Understanding age-related tauopathy is of critical importance in the context of diagnosis and staging of all the tauopathies given its extremely high prevalence and importance as a comorbidity in essentially all studies evaluating tauopathy.

There has been controversy surrounding the PART consensus criteria since their introduction $[9,17]$, and there have been a substantial number of recent clinicopathological studies focused on understanding this pathological presentation $[4-6,27,31,34,50,51,60]$. Given the close clinical and neuropathological similarities between PART and AD such that historically the two entities were classified together, accumulating evidence has highlighted differences. Clinically, the average age is higher for individuals who have PART than those with $\mathrm{AD}$ and patients with PART are more often female [33]. Patients with PART pathology are more often cognitively normal, but a subset have mild cognitive impairment or amnestic dementia, and this correlates with p-tau severity [15]. Among symptomatic individuals with a neuropathological diagnosis of PART, nearly half had been clinically diagnosed with AD compared with $86 \%$ of those with autopsy-confirmed $\mathrm{AD}$, indicating that despite diagnostic uncertainty, clinicians recognize differences between the two [59]. One retrospective study identified other factors including depression, Braak stage, and history of stroke, as independent predictors of cognitive impairment [5]. Another found that those with PART had a sparing of semantic memory compared to those with $\mathrm{AD}$, suggesting that there is a distinct difference in clinical presentation [6]. Longitudinal analyses found that subjects with PART have a significantly slower clinical decline after becoming symptomatic than those with $\mathrm{AD}$ across multiple neuropsychological domains [60].

One limitation of most published studies on PART is that they rely on retrospective analysis of previously collected datasets (e.g., the National Alzheimer's Coordinating Center database, NACC) with predefined neuropathological measures that may not fully capture all the clinically relevant features [43]. Further, findings might not be generalizable to other populations, and a lack of uniform analysis and quantitation might lead to bias. Critically, the Braak staging system was specifically developed for assessment of tau pathology in the context of $\mathrm{AD}$, and has not been rigorously tested in amyloidnegative subjects, so the extent to which it is valid for staging p-tau pathology in PART is unclear. Additionally, the Braak stage represents a hierarchical progression of the regional spread of neurofibrillary tangles, but does not directly measure the severity or burden of p-tau, but this has been incorporated into some operationalized frameworks [2]. Because the pathology in PART generally remains predominantly in the medial temporal lobe, this hierarchical pathoanatomical system may sub-optimally measure severity of the disease. There are numerous approaches to assessing lesion burden of p-tau and other pathologies $[8,26,28,29,38,39,42]$, including cell counting and stereology $[3,4,11,19,25,64]$. While each of these approaches have intrinsic advantages, they are limited in that they are labor intensive and for this reason and others, these methods have not been widely adopted in neuropathology laboratories $[18,62]$. One approach that may have potential to better assess p-tau in PART is using computer-assisted quantitative morphometrics on 
digital whole slide images, which may be well suited for staging PART.

Here, we studied a cohort of autopsy-confirmed subjects with PART, enabling us to reexamine how tau pathology manifests in PART. We compared Braak staging with computer-assisted quantitative measures of $\mathrm{p}$-tau burden, and used logistic regression to assess their contribution to cognitive impairment. Using this cohort, we were able to explore critical co-morbid pathologies (e.g., cerebrovascular disease), and further assess neuropathological changes that are not available in existing publicly available datasets, including atrophy and ARTAG.

\section{Methods}

\section{Patient samples}

Formalin-fixed paraffin embedded (FFPE) tissue from the frontal cortex and hippocampus as well as freshfrozen tissue from frontal cortex were derived from autopsy brains from a subset of individuals from a previously described collection [61]. Specifically, the cohort included cases from the Oregon Health Sciences University (Portland, OR, USA), Banner Sun Health Research Institute (Sun City, AZ, USA), Emory (Atlanta, GA, USA), Northwestern (Evanston, IL, USA), the University of Pennsylvania (Philadelphia, PA, USA), University of Pittsburgh (Pittsburgh, PA, USA), University of Texas Southwestern Medical Center (Dallas, TX, USA), and the Medical University of Vienna (Vienna, Austria). Clinical inclusion criteria included being cognitively normal or having a diagnosis of mild cognitive impairment (MCI) or dementia with a recorded clinical dementia rating (CDR), Mini-Mental State Examination (MMSE), or postmortem clinical chart review CDR score within two years of death $[20,45]$. CDR and MMSE scores were used to assign subjects into either cognitively normal or cognitively impaired groups. Individuals who had a CDR score of 0.5 or above or MMSE score below 26 were considered to be cognitively impaired while subjects with a CDR score of 0 or MMSE score 26 or above were considered cognitively normal [37]. If an individual had both MMSE score and CDR score, the most recent score was used, and if both scores were given on the same date, the CDR score was used.

Comprehensive neuropathological assessments were performed at the contributing institutions. Neuropathological criteria for PART included (1) cases that had a Braak stage of 0-IV and (2) Consortium to Establish a Registry for Alzheimer's Disease (CERAD) neuritic plaque severity score of $0[8,42]$. Neuropathological exclusion criteria consisted of other neurodegenerative diseases including $\mathrm{AD}$, Lewy body disease, progressive supranuclear palsy (PSP), corticobasal degeneration
(CBD), chronic traumatic encephalopathy (CTE), Pick disease, Guam amyotrophic lateral-sclerosis-parkinsonism-dementia, subacute sclerosing panencephalitis, globular glial tauopathy, and hippocampal sclerosis. Data pertaining to Braak stage, CERAD, Lewy body pathology (incidental), cerebrovascular disease, infarcts (vascular brain injury), microinfarcts, and argyrophilic grains, were derived from neuropathologic studies performed at respective centers. Incidental Lewy body pathology was defined as the presence of rare to sparse Lewy bodies (as assessed at the providing center) in the absence of movement disorder. The presence of aging-related tau astrogliopathy (ARTAG) was determined on p-tau immunohistochemical stains described below [36].

\section{Atrophy score}

Given that no widely accepted validated system for assessing hippocampal atrophy on human brain sections exists, we devised a semiquantitative scoring system and applied it to low power images of hematoxylin \& eosinstained sections counterstained with Luxol fast blue. We defined atrophy severity as the magnitude of ventricular dilatation (hydrocephalus ex vacuo) relative to the size of the hippocampal formation. If there was no apparent ventricular dilatation or atrophy, then a score of 0 was assigned. If there was appreciable atrophy, but the dorsoventral height of the ventricle was less than the height of the thickest section of CA1, then a score of 1 (mild) was assigned. If the magnitude of ventricular dilatation exceeded the thickness of CA1, then a score of 2 (moderate) was given. If the total area of the ventricle area was greater than the area of the hippocampus proper, a score of 3 (severe) was assigned. This score was derived only in the subset of cases where the entire temporal horn of the lateral ventricle was available included in the provided section $(n=24)$.

\section{Immunohistochemistry}

Immunohistochemistry (IHC) and hematoxylin \& eosin (H\&E) stains were performed on FFPE Sections. $(5 \mu \mathrm{m})$ that were prepared from blocks of hippocampus and frontal cortex for supplemental neuropathological analyses (see below). Sections mounted on positively charged slides were dried overnight at room temperature. IHC was performed on a Leica Bond III automated stainer, according to the manufacturer's protocols (Leica Microsystems, Buffalo Grove, IL, USA). IHC was performed using antibodies to hyper-phosphorylated tau (p-tau, AT8, 1:1000, Fisher Scientific, Waltham, MA) and beta-amyloid (A $\beta, 6 E 10,1: 1000$, Covance, Princeton, N), USA). $A \beta$ stains were confirmed to be negative to ensure that there were no neuritic or diffuse plaques present 
(CERAD score of 0 ) for all cases. For each set of slides stained, a known severe AD case was included as a batch control.

\section{Computer-assisted morphometric analysis}

Whole slide images (WSI) were prepared from glass slides that were scanned using an Aperio CS2 (Leica Biosystems, Wetzlar Germany) digital slide scanner. Quantitative analysis of the tau burden was performed in selected regions in the hippocampi using the following methodology; WSI were neuroanatomically segmented using Aperio ImageScope software into the hippocampus proper (i.e., dentate, cornu ammonis, and subiculum) and the adjacent cortex that we termed the entorhinal region, which variably includes posterior portions of the parahippocampal gyrus with remnants of the (trans-)entorhinal region or lingual gyrus. Staining was measured in these areas separately and together using a modified version of the Aperio positive pixel count (Version 9) based on the intensities of the positive control sample in each batch to determine the area of immunoreactivity. Data were normalized using the number of positive pixel counts to the total area creating a $0-1 \mathrm{p}$-tau burden scale.

\section{Genetic analysis}

High-throughput isolation of DNA was performed using the MagMAX DNA Multi-Sample Ultra 2.0 Kit on KingFisher Flex robotic DNA isolation system (Thermofisher, Waltham, MA). 20-40 mg of fresh frozen brain tissue were placed into a deep-well plate and treated with 480 ul of Proteinase K mix (Proteinase K, Phosphate Buffered Saline [pH 7.4], Binding Enhancer) and incubated overnight at $65{ }^{\circ} \mathrm{C}$ at $800 \mathrm{rpm}$ on a shaking plate. Genomic DNA was isolated and purified using magnetic particles. DNA quality control was performed using a nanodrop spectrophotometer (concentration $>50 \mathrm{ng} / \mathrm{ul}, 260 / 280$ ratio 1.7-2.2). Genotyping was performed using single nucleotide polymorphism (SNP) microarrays (Infinium Global Screening Array v2.4. or the Infinium OmniExpress-24, Illumina, San Diego CA). Raw genotype files were converted to PLINK-compatible files using GenomeStudio software (Illumina, San Diego CA). MAPT haplotype was determined using the rs8070723 $\mathrm{H} 2$ tagging SNP. APOE genotype was provided by the collaborating center. For analyses, the $A P O E$ status was collapsed into a binary variable of the presence or absence of APOE $\varepsilon 4$.

\section{Statistical analysis}

All statistical tests were performed using the statistical software Statistical Package for the Social Sciences (SPSS) (IBM, Chicago, Il). Data was visualized using the ggplot2 package in project $\mathrm{R}$ or Excel (Microsoft, Redmond, Washington). Binary measurements (yes/no) were created for pathological, clinical, demographic, and genetic variables. Specifically, variables were extracted from the pathological diagnosis and binary measurements (yes/no) were created for the following variables: argyrophilic grains, Lewy body pathology (incidental), cerebrovascular disease, and infarcts (vascular brain injury). Additionally, the same process was done for clinical variables: history of psychiatric illness and education (for this study, defined as at least some college).

Descriptive statistics were used to identify differences between the cognitively normal and cognitively impaired PART groups for clinical, pathological, and genetic variables. Differences were detected using $X^{2}$ tests or exact $\chi^{2}$ if any cell size included $<5$ participants. A t-test was performed to determine if age differed significantly between normal and cognitively impaired groups. Next, an unadjusted binary logistic regression was performed to determine what genetic, clinical, and pathological variables were associated with being cognitively impaired within our PART cohort. Lastly, a multivariable model was created to determine what extent Braak NFT stage and the computer-assisted morphometrics were able to predict cognitive impairment in PART when adjusting for age. Statistical significance was determined if $\alpha<0.05$. Not all data was available on the subjects.

\section{Results}

One hundred seventy-four neuropathologically confirmed amyloid-negative subjects were included in this study (Table 1, Fig. 1). The overall mean age was 83.2 with a range of 52.9-105.1 years. Of these, 124 subjects (mean age 81.0, range $=52.9-102.4$ ) had no cognitive impairment and 50 (mean age 88.3, range $=69.8-105.1$ ) had some degree of cognitive impairment, with either mild cognitive impairment (MCI) or dementia. The majority of subjects who were cognitively impaired were $80+$ years of age (Fig. 2). The Braak NFT stage ranged from 0 to IV with the majority of cognitively impaired subjects having a Braak NFT score of II to IV. A higher percentage of females had cognitive impairment (62.0\%) compared to those who were cognitively normal (49.2\%).

We observed several differences among subjects with cognitive impairment compared to those who were cognitively normal. First, cognitively impaired PART subjects were more likely to be older (age of testing 81.0 vs. 88.3 , $p<0.0001)$, have cerebrovascular disease $(42.0 \%$ vs. $4.8 \%$, $p<0.0001)$ and have hippocampal age-related tau astrogliopathy (ARTAG; $38.3 \%$ vs. $21.6 \%, p<0.05$ ) compared to cognitively normal subjects (Table 1 ). However, education, history of psychiatric illness, argyrophilic grains, incidental Lewy body pathology, infarcts, presence of an $A P O E \& 2$ allele, presence of $A P O E \varepsilon 4$ allele, and MAPT 
Table 1 Patient data

\begin{tabular}{|c|c|c|c|c|}
\hline & \multirow[b]{2}{*}{ Overall } & \multicolumn{2}{|l|}{ Cognitive status } & \multirow[b]{2}{*}{$p$} \\
\hline & & Normal & Impaired* & \\
\hline \multicolumn{5}{|l|}{ Demographics } \\
\hline Average age at testing (range) & $83.2(52.9-105.1)$ & $81.0(52.9-102.4)$ & $88.3(69.8-105.1)$ & $<0.0001$ \\
\hline Total (Male / Female) & $174(82 / 92)$ & $124(63 / 61)$ & $50(19 / 31)$ & $0.126^{* * *}$ \\
\hline \multicolumn{5}{|l|}{ Age at last visit (\%) } \\
\hline$<60$ & $7(4.0)$ & $7(5.6)$ & $0(0.0)$ & \\
\hline $60-69$ & $15(8.6)$ & $14(11.3)$ & $1(1.7)$ & \\
\hline $70-79$ & $33(19.0)$ & $30(24.2)$ & $3(5.2)$ & \\
\hline $80-89$ & $76(43.7)$ & $45(36.3)$ & $31(53.4)$ & \\
\hline $90+$ & $51(29.3)$ & $28(22.6)$ & $23(39.7)$ & \\
\hline Education, at least some college (\%) & $32(18.4)$ & 15 (78.9) & $17(77.3)$ & 0.89 \\
\hline History of psychiatric illness (\%) & $45(25.9)$ & $29(31.9)$ & $17(45.9)$ & 0.13 \\
\hline \multicolumn{5}{|l|}{ Neuropathological data } \\
\hline Argyrophilic grains & $32(18.4)$ & $12(9.7)$ & $10(20.0)$ & 0.06 \\
\hline Lewy body pathology (incidental) & $16(9.2)$ & $11(8.9)$ & $5(10.0)$ & 0.82 \\
\hline Cerebrovascular disease $e^{* *}$ & $27(15.5)$ & $6(4.8)$ & $21(42.0)$ & $<0.0001$ \\
\hline Infarcts (vascular brain injury) & $37(21.3)$ & $24(19.4)$ & $13(26.0)$ & 0.33 \\
\hline Hippocampus ARTAG positive (\%) & $43(24.7)$ & $25(21.6)$ & $18(38.3)$ & 0.03 \\
\hline \multicolumn{5}{|l|}{ Genetic data } \\
\hline Presence of $\geq 1 A P O E$ \&4 allele & $22(12.6)$ & $16(12.9)$ & $6(11.3)$ & 0.77 \\
\hline Presence of $\geq 1 A P O E$ \&2 allele & $46(26.4)$ & $27(21.8)$ & $19(35.8)$ & 0.06 \\
\hline Presence of $\geq 1 \mathrm{MAPT} \mathrm{H} 2$ & $59(33.9)$ & $42(36.2)$ & $17(36.2)$ & 1 \\
\hline
\end{tabular}

* Mild cognitive impairment or dementia, ${ }^{* *}$ excluding cerebral amyloid angiopathy, ${ }^{* * *}$ Male sex, significant values in bold (Chi squared test)

haplotype status did not significantly affect cognitive status ( $p>0.05$ for all conditions).

In our main unadjusted analysis, we assessed the extent to which a series of clinical, neuropathological, and genetic variables predicted cognitive impairment in our PART cohort (Table 2). We found that age and cerebrovascular disease were the strongest predictors of cognitive impairment $(p<0.0001$ for both cases). ARTAG and hippocampal atrophy were also significant predictors, but to a lesser extent $(p<0.05$ for both cases). There were more reported men and subjects with a history of psychiatric illness, argyrophilic grains, incidental Lewy body pathology, infarcts, and microinfarcts in the cognitively impaired PART group, however none of these predictors was significantly different ( $p>0.05$ for all conditions). APOE \&4 (at least $1 \varepsilon 4$ allele) was reported more in the cognitively normal PART group but did not reach significance. Braak NFT stage significantly predicted cognitive impairment $(p<0.05)$. Additionally, the computer-assisted morphometrics in the entorhinal region, hippocampus proper, and the combined region were significantly associated with cognitive impairment $(p=0.0001$, Fig. $3 a-c$,
Table 3). Lastly, when the Braak NFT stage was correlated with computer-assisted morphometrics in the combined region $(p<0.001)$, there was a high degree of variability between the Braak NFT stage and the computer-assisted combined region morphometrics (Fig. 3d).

Finally, using a multivariable model, we assessed what features predicted cognitive impairment when controlling for age. In this adjusted analysis, we found cerebrovascular disease remained significantly associated with cognitive impairment $(p<0.00001)$. We also found that computer-assisted morphometrics used to capture p-tau burden in the hippocampus proper and combined region were significantly associated with cognitive impairment in PART $(p<0.05$ for both cases). However, the computer-assisted morphometrics in the entorhinal region were not associated with cognitive impairment yet there was a trend toward statistical significance $(p=0.07)$. The Braak NFT stage was not able to predict cognitive impairment when controlling for age $(p=0.98$, Table 3 , Fig. 4). Additionally, we collapsed Braak NFT stage to low $(0$, I, II) and high (III, IV) and still did not observe statistically significant differences (data not shown). 


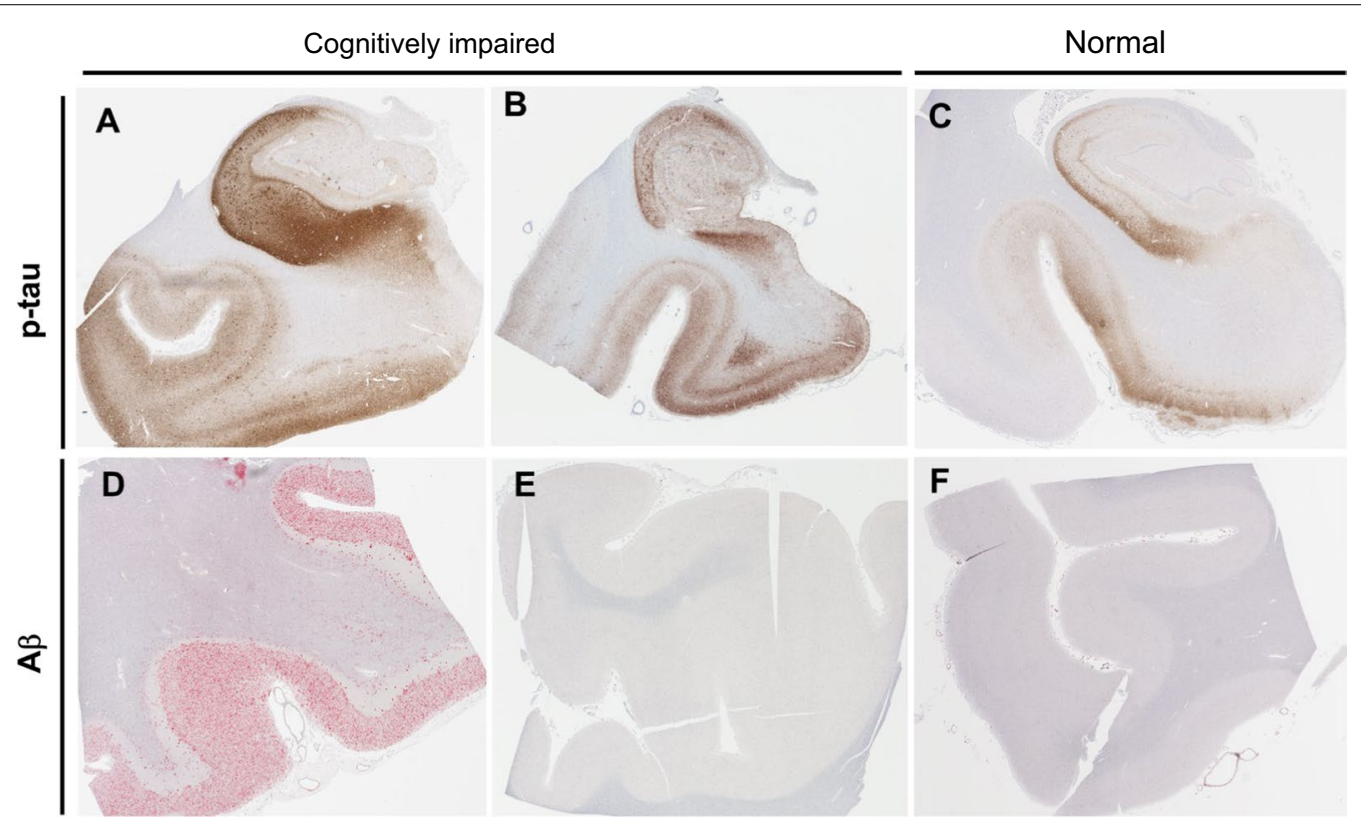

Fig. 1 Comparison of amyloid and tau pathology in primary age-related tauopathy (PART) versus Alzheimer disease (AD). a Immunohistochemical staining using antisera to hyperphosphorylated tau in an AD brain shows marked hyperphosphorylated tau ( $p$-tau)-containing neurofibrillary tangles (NFT) in the hippocampus which extends past the collateral sulcus into the parahippocampal gyrus and other neocortical regions. b, $\mathbf{c}$ Subjects with mild to severe PART have elevated p-tau levels in the hippocampus predominantly restricted to the medial temporal lobe. $\mathbf{d}, \mathbf{e}, \mathbf{f}$ Subjects with AD neuropathologic change have abundant A $\beta$-containing plaques in neocortical structures, whereas those with PART have sparse or none. These neuropathologic changes in AD and PART are seen in association with varying degree of cognitive impairment ranging from cognitively normal to demented
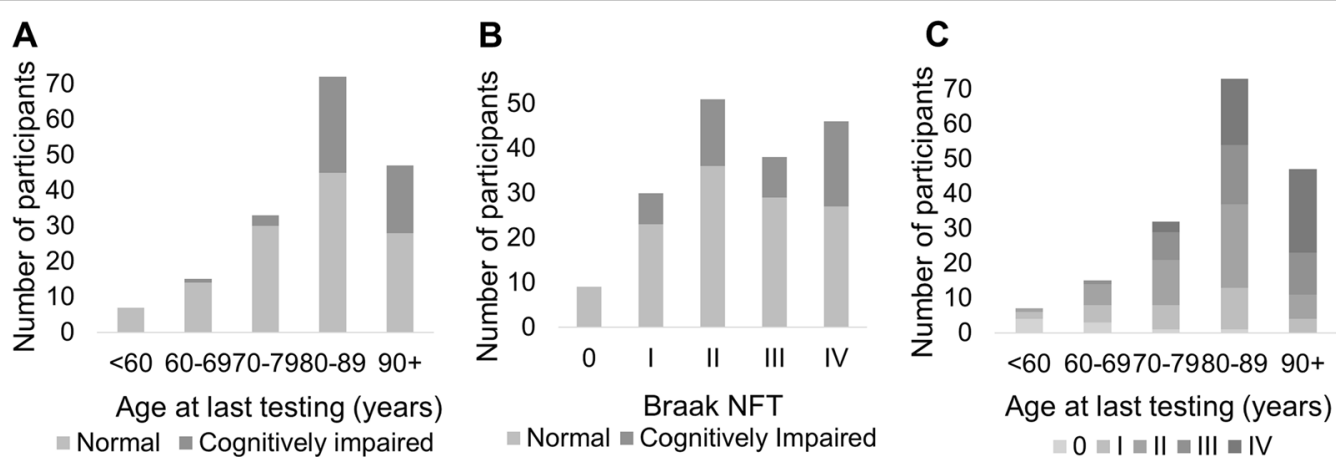

Fig. 2 Distribution of age, Braak neurofibrillary tangle (NFT) stage and cognitive status. a The number of normal and cognitively impaired subjects across the age spectrum. $\mathbf{b}$ The number of cognitively normal and impaired subjects by Braak stage. $\mathbf{c}$ The number of subjects across the aging spectrum by Braak stage

\section{Discussion}

Since the neuropathological criteria for PART were proposed, the terminology has been widely adopted, but controversy persists, especially around its relationship to Alzheimer disease (AD). Delineating the histological/cellular features that are associated with cognitive impairment in PART is critical for advancing our understanding of the pathology and determining the extent to which it overlaps with $\mathrm{AD}$. The fact that subjects with PART, as with $\mathrm{AD}$ neuropathologic change, can range in their cognitive status from normal to demented, raises the question as to whether cognitive reserve/resilience plays a role or alternatively whether we are not adequately capturing the relevant features, such as common comorbidities or other factors. This study, by using a large autopsy cohort with multivariate analyses, directly addresses 
Table 2 Unadjusted odds of being cognitively impaired

\begin{tabular}{|c|c|c|c|}
\hline & OR & $95 \% \mathrm{Cl}$ & $p$ value \\
\hline \multicolumn{4}{|l|}{ Characteristic } \\
\hline Age, at testing & 1.08 & $1.04-1.13$ & $<0.0001$ \\
\hline Education, y & 0.87 & $0.67-1.12$ & 0.28 \\
\hline Sex & 1.69 & $0.86-3.30$ & 0.13 \\
\hline APOE (at least 1 \&4 allele) & 0.988 & $0.36-2.70$ & 0.98 \\
\hline History of psychiatric diagnosis & 1.82 & $0.83-3.98$ & 0.14 \\
\hline $\begin{array}{l}\text { Aging-related tau astrogliopathy } \\
\text { (ARTAG) }\end{array}$ & 2.26 & $1.08-4.72$ & 0.03 \\
\hline Argyrophilic grains & 2.33 & $0.94-5.82$ & 0.07 \\
\hline Lewy body pathology (incidental) & 1.14 & $0.38-3.47$ & 0.82 \\
\hline Cerebrovascular disease $e^{*}$ & 14.24 & $5.27-38.48$ & $<0.0001$ \\
\hline Infarcts (vascular brain injury) & 1.46 & $0.68-3.17$ & 0.33 \\
\hline Microinfarcts & 1.05 & $0.43-2.59$ & 0.91 \\
\hline Hippocampal atrophy & 5.32 & $1.04-27.09$ & 0.04 \\
\hline Braak NFT stage & 1.37 & $1.03-1.83$ & 0.03 \\
\hline \multicolumn{4}{|c|}{ Computer-assisted p-tau (AT8) burden (positive pixel counts) } \\
\hline Entorhinal region & 1.90 & $1.31-2.75$ & 0.001 \\
\hline Hippocampus proper & 2.17 & $1.48-3.20$ & $<0.0001$ \\
\hline $\begin{array}{l}\text { Entorhinal region \& Hippocampus } \\
\text { proper }\end{array}$ & 2.12 & $1.44-3.11$ & $<0.0001$ \\
\hline
\end{tabular}

*Excluding cerebral amyloid angiopathy, significant values in bold (logistic regression)

these critical questions. The goal was to leverage our collection of post-mortem PART brains to characterize the clinical, pathological, and genetic features that are associated with cognitive impairment in PART. Additionally, we sought to compare Braak stage with pathology burden measures derived from $\mathrm{p}$-tau immunohistochemistry that quantifies severity independently of neuroanatomical vulnerability. To overcome intra-center variability in tau pathology measures, we reassessed each case histologically to maximize accuracy. This study design, which included the entire spectrum of Braak 0-IV treated PART pathology as a ubiquitous quantitative trait allowing us to examine the continuum of $\mathrm{p}$-tau burden in the absence of amyloid deposition.

We found that all of our PART definite cases had p-tau restricted mainly to the MTL (Braak NFT stage $<$ IV), which is consistent with and supports other previous studies investigating PART [4, 15, 32]. Cases ranged in cognitive impairment with the majority of subjects being cognitively normal, and consistent with prior data, the PART subjects tended to be older than individuals with AD $[15,59]$. The results of our study confirm those of previous autopsy studies showing that cerebrovascular disease predicts cognitive impairment in PART, even when adjusted for age $[5,48]$. Interestingly, we did not see a strong correlation between cognitive impairment and microinfarcts, while others have shown a correlation with cognition in the oldest old [12]. We did however, find novel, unreported associations of increased age, hippocampal atrophy, and ARTAG with cognitive impairment in our PART definite cohort. Similar to what has been reported by those utilizing the NACC database, our results verify those with a higher Braak NFT stage are associated with more rapid cognitive decline [31].

While these associations have yet to be reported in PART, there are numerous studies showing that age, atrophy, and ARTAG may be associated with cognitive impairment [7, 21, 30, 49, 52]. Surprisingly, we did not see increased odds of the Braak NFT stage being associated with cognitive impairment when controlling for age as has been reported in other studies [5]. However, we did find that using computer-assisted morphometrics to assess p-tau burden in the entorhinal region, hippocampus, and combined region was able to significantly predict cognitive impairment, similar to other studies $[1,11]$. While Braak NFT staging is the most widely employed approach for assessing p-tau, it is limited in that it primarily focuses on regionality and not disease burden [23]. Other studies have employed both manual and computer assisted quantitative approaches that may capture aspects of pathological features with more power $[22,24,55]$. However, a majority of these approaches focuses on $\mathrm{AD}$ which may not be relevant in the context of PART, where p-tau pathology does not progress in the same hierarchical manner proposed by Braak in AD [8, 14]. Hence this study highlights several new methodologies to assess $\mathrm{p}$-tau burden, which our results suggest to be a more accurate predicator of clinical symptomology in those with PART. However, until digital neuropathology becomes more widespread, semi-quantitative assessment might be a better approach to staging PART in clinical neuropathology practice, but further validation is required.

In addition to assessing p-tau burden, we also examined the effect of $A P O E$ status in PART as a predictor for cognitive impairment. $A P O E \varepsilon 4$ has been strongly suggested as an important predictor of cognitive decline in $\mathrm{AD}$ while $A P O E \varepsilon 2$ has been shown to be protective [13, $16,53,57]$. However, many of these studies have been performed in $\mathrm{AD}$ cohorts, and in aging cohorts there has been evidence suggesting the $\varepsilon 4$ allele is not a risk factor for cognitive impairment [56]. Our data agree with that reported by Small et al. as we did not see an association with $A P O E \& 4$ and cognitive impairment, which might be explained by the fact that we studied a pathologically confirmed amyloid-negative cohort. Recent work has suggested that $A P O E$ may exacerbate tau pathology independently of amyloid deposition [54]. Here, we failed to detect an association of cognitive impairment in PART with the MAPT H1 haplotype; future larger studies 

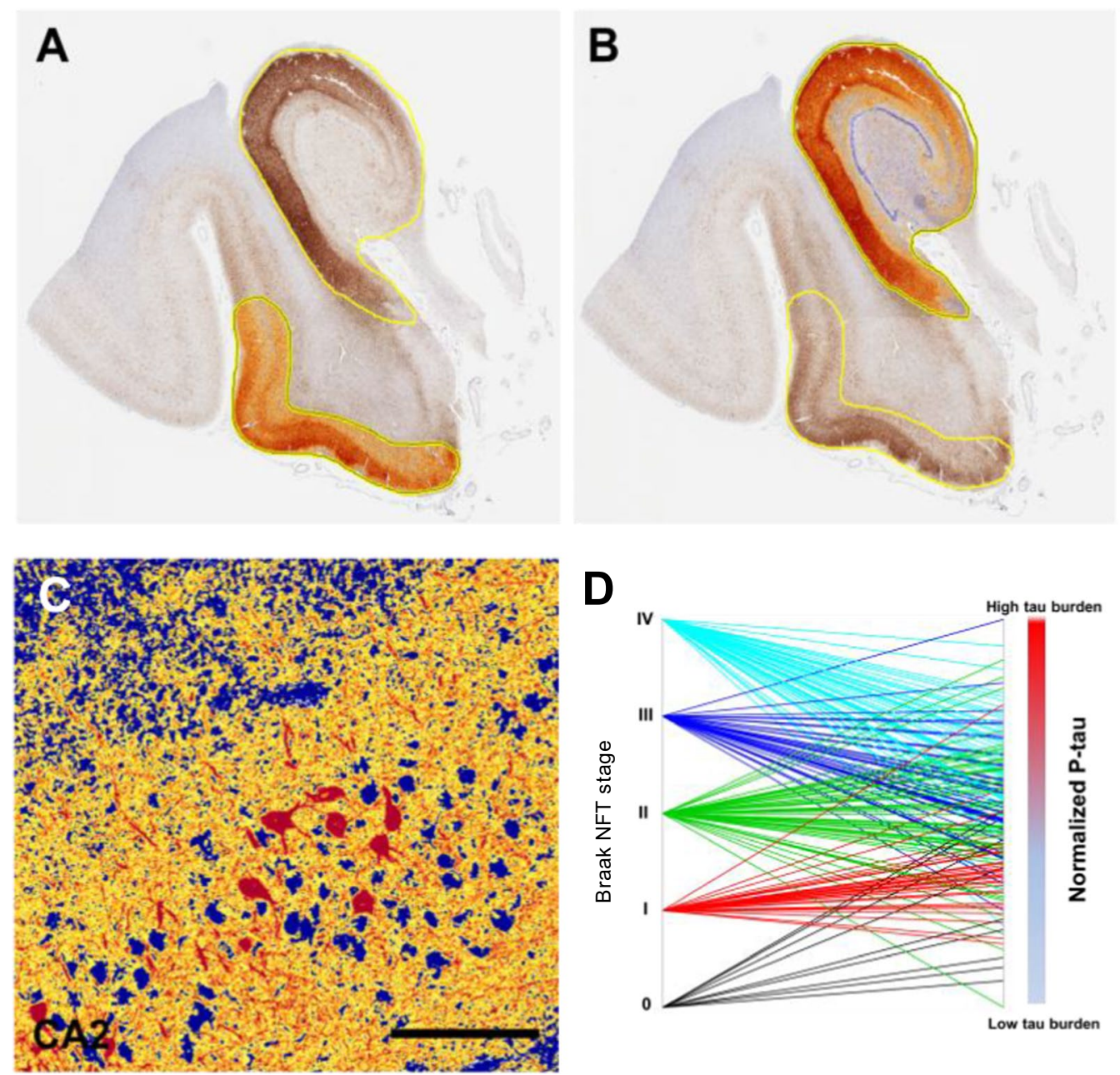

Fig. 3 Computer-assisted morphometrics to assess pathological tau burden. a, b Quantitative assessment of hyperphosphorylated tau ( $p$-tau) burden was performed on whole slide images of the hippocampus stained for p-tau (AT8) using immunohistochemistry. Positive pixel counts were determined in two regions (hippocampus proper and entorhinal region). Results were normalized to the total area assessed. A third summary score of the total p-tau burden of the medial temporal lobe was calculated by summing positive pixels in both. $\mathbf{c}$ High power image shows high intensity in red, medium intensity in yellow and negative staining in blue. $\mathbf{d}$ Parallel plot showing the relationship between Braak stage and the computer morphometric quantification of p-tau using the normalized medial temporal lobe (hippocampus and entorhinal region). Scale bar $=150 \mu m$

Table 3 Odds of being cognitively impaired at death, adjusted

\begin{tabular}{lllc}
\hline & OR & $\mathbf{9 5 \% ~ C l}$ & $p$ value \\
\hline Cerebrovascular disease & 11 & $1.47-3.41$ & $<0.0001$ \\
Braak NFT stage & 1.01 & $0.72-1.41$ & 0.98 \\
P-tau burden (computer-assisted AT8 IHC positive pixels) & & & 0.07 \\
Entorhinal region & 1.46 & $0.97-2.20$ & $\mathbf{0 . 0 2}$ \\
Hippocampus & 1.66 & $1.07-2.57$ & $\mathbf{0 . 0 3}$ \\
Entorhinal region \& hippocampus & 1.62 & $1.06-2.49$ & \\
\hline
\end{tabular}

Significant values in bold (logistic regression) 


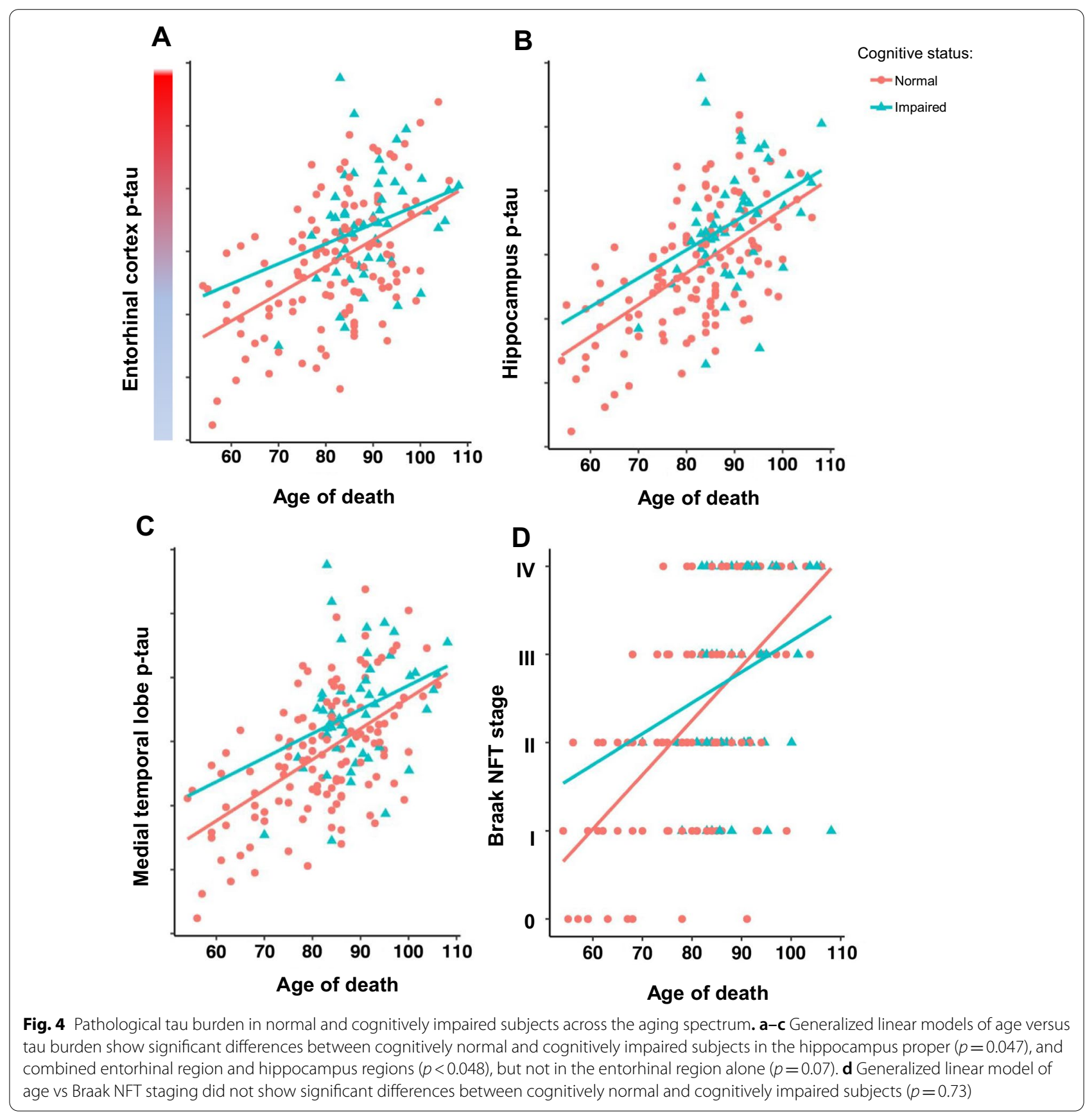

with more statistical power are required to delineate the genetic architecture of PART.

This study had notable limitations. There was a relatively small number of subjects in the cognitively impaired PART group $(n=50)$, which may weaken our power to predict cognitive impairment. Additionally, because a majority of our subjects were not from longitudinally studied prospective cohorts, we were unable to obtain certain lifestyle variables, such as actual years of education and concussion history, which could potentially significantly affect our model. However, given that diagnosing PART pre-mortem is currently challenging, it would be impractical to create such a prospective cohort. One important consideration not directly addressed is limbic-predominant age-related TDP43 encephalopathy neuropathologic changes (LATE-NC) [47]. LATE-NC is a potential driver of cognitive changes in our cohort given its high frequency in the elderly population, with up to 
$25 \%$ of subjects in community-based autopsy cohorts having sufficient LATE-NC burden to be cause cognitive impairment [47]. Given that PART commonly presents with co-morbidities including LATE-NC, the relationship between TDP-43 proteinopathy and PART should be examined more closely [34, 58, 63]. Additionally, the association we observed with ARTAG and cognitive status might be only due to collinearity between p-tau severity and ARTAG, with p-tau probably the driving pathology and the ARTAG association being significant because of its potential dependence on p-tau. Lastly, our study was limited to pathology of the medial temporal lobe and frontal cortex. A more exhaustive study would have incorporated a greater number of brain regions to more extensively address other potential tau-related pathologies.

In summary, our findings are consistent with the hypothesis that PART is an amyloid-independent tauopathy, primarily affecting the medial temporal lobe, which can present with cognitive impairment. Several demographic and neuropathological variables including age, ARTAG, cerebrovascular disease, hippocampal atrophy, Braak NFT stage, and p-tau computer assessments were significantly associated with cognitive impairment in our PART cohort. The Braak NFT stage was not a significant predictor of cognitive impairment when controlling for age, while the computer-assistant morphometrics were. These data strongly suggest that neuroanatomical staging used in AD may not be as relevant to PART given the pathology minimally spreads beyond the medial temporal lobe. Novel techniques to measure p-tau burden can further our understanding of PART pathology and associated clinical and genetic features.

\section{Acknowledgements}

This work was supported by the National Institutes of Health [R01 AG054008, R01 NS095252, R01 AG060961, and R01 NS086736 to J.F.C., F32 AG056098 and P30 AG066514 to K.F., R01 AG062348 to J.F.C., A.M., and D.D., P30 AG010124, P01 AG017586 and U19 AG062418 to J.Q.T., P30AG072979 and P01AG066597 to E.B.L., R01 AG066152 to C.T.M., P30 AG066468 to J.K., P50 AG005138, P30 AG066514, and 75N95019C00049 to V.H., U24 NS072026 and P30 AG019610 to T.B., P30 AG013854 to E.B., P30 NS055077 and P50 AG025688 to M.G., P30 AG08017 to R.W. and U54 NS115266 to A.M.], the Alzheimer's Association [NIRG-15-363188 to J.F.C.], the Tau Consortium, Genentech/Roche, David \& Elsie Werber, Alexander Saint-Amand Fellowship, J.M.R. Barker Foundation, The McCune Foundation, and the Winspear Family Center for Research on the Neuropathology of Alzheimer Disease, The Arizona Department of Health Services, and the Michael J. Fox Foundation for Parkinson's Research. G.G.K. is supported by the Rossy Foundation and by the Safra Foundation. The authors would also like to acknowledge Ping Shang, HT(ASCP) QIHC and Jeff Harris, HTL(ASCP) for histologic and immunohistochemical preparations, and Chan Foong, M.S., for preparation of whole slide image.

\section{Declarations}

\section{Competing interests}

The authors declare that they have no conflicts of interest.

\section{Author details}

${ }^{1}$ Department of Pathology, Nash Family Department of Neuroscience, Ronald M. Loeb Center for Alzheimer's Disease, Icahn School of Medicine At Mount Sinai, Friedman Brain Institute, Neuropathology Brain Bank \& Research CoRE, 1 Gustave L. Levy Place Box 1194, New York, NY 10029, USA. ${ }^{2}$ Department of Pathology and Laboratory Medicine and The Glenn Biggs Institute for Alzheimer's and Neurodegenerative Diseases, UT Health San Antonio, San Antonio, TX, USA. ${ }^{3}$ Ultragenyx Pharmaceuticals, Novato, CA, USA. ${ }^{4}$ Banner Sun Health Research Institute, Sun City, AZ, USA. ${ }^{5}$ Department of Pathology, Northwestern Cognitive Neurology and Alzheimer Disease Center, Northwestern University Feinberg School of Medicine, Chicago, IL, USA. ${ }^{6}$ Department of Pathology and Laboratory Medicine, Emory University School of Medicine, Atlanta, GA, USA. ${ }^{7}$ Departments of Psychiatry and Neuroscience, Alzheimer's Disease Research Center, Icahn School of Medicine At Mount Sinai, New York, NY, USA. ${ }^{8}$ JJ Peters VA Medical Center (MIRECC), Bronx, NY, USA. ${ }^{9}$ Department of Neurology, Perelman School of Medicine, Penn FTD Center, Center for Neurodegenerative Disease Research, University of Pennsylvania, Philadelphia, PA, USA. ${ }^{10}$ Department of Neuroscience, Mayo Clinic, Jacksonville, FL, USA. ${ }^{11}$ Department of Pathology, VA Medical Center \& Boston University School of Medicine, Boston, MA, USA. ${ }^{12}$ Center for Neurodegenerative Disease Research, Department of Pathology and Laboratory Medicine, Perelman School of Medicine, University of Pennsylvania, Philadelphia, PA, USA. ${ }^{13}$ Department of Pathology, Oregon Health Sciences University, Portland, OR, USA. ${ }^{14}$ Laboratory Medicine Program, Krembil Brain Institute University Health Network Toronto Ontario, Ontario, Canada. ${ }^{15}$ Department of Laboratory Medicine and Pathobiology, Tanz Centre for Research in Neurodegenerative Disease, University of Toronto, Toronto, ON, Canada. ${ }^{16}$ Institute of Neurology, Medical University of Vienna, Vienna, Austria. ${ }^{17}$ Department of Pathology, University of Pittsburgh Medical Center, Pittsburgh, PA, USA. ${ }^{18}$ Department of Neurology, Oregon Health \& Science University, Portland, USA. ${ }^{19}$ Neuropathology Laboratory, Department of Pathology, University of Texas Southwestern Medical Center, Dallas, USA. ${ }^{20}$ Department of Pathology and Laboratory Medicine, Translational Neuropathology Research Laboratory, Perelman School of Medicine at the University of Pennsylvania, Philadelphia, PA, USA.

Received: 2 June 2021 Accepted: 16 July 2021

Published online: 05 August 2021

\section{References}

1. Abner EL, Neltner JH, Jicha GA, Patel E, Anderson SL, Wilcock DM, Van Eldik LJ, Nelson PT (2018) Diffuse amyloid-beta plaques, neurofibrillary tangles, and the Impact of APOE in elderly persons' brains lacking neuritic amyloid plaques. J Alzheimers Dis 64:1307-1324. https://doi.org/10.3233/ JAD-180514

2. Alafuzoff I, Arzberger T, Al-Sarraj S, Bodi I, Bogdanovic N, Braak H, Bugiani O, Del-Tredici K, Ferrer I, Gelpi E et al (2008) Staging of neurofibrillary pathology in Alzheimer's disease: a study of the BrainNet Europe Consortium. Brain Pathol 18:484-496. https://doi.org/10.1111/j.1750-3639.2008. 00147.x

3. Attems J, Neltner JH, Nelson PT (2014) Quantitative neuropathological assessment to investigate cerebral multi-morbidity. Alzheimers Res Ther 6: ARTN 85. https://doi.org/10.1186/s13195-014-0085-y

4. Bell WR, An Y, Kageyama Y, English C, Rudow GL, Pletnikova O, Thambisetty M, O'Brien R, Moghekar AR, Albert MS et al (2019) Neuropathologic, genetic, and longitudinal cognitive profiles in primary age-related tauopathy (PART) and Alzheimer's disease. Alzheimers Dement 15:8-16. https://doi.org/10.1016/j.jalz.2018.07.215

5. Bennett DA, Buchman AS, Boyle PA, Barnes LL, Wilson RS, Schneider JA (2018) Religious orders study and rush memory and aging project. Adv Alzh Dis 6:159-187. https://doi.org/10.3233/978-1-61499-876-1-159

6. Besser LM, Crary JF, Mock C, Kukull WA (2017) Comparison of symptomatic and asymptomatic persons with primary age-related tauopathy. Neurology 89:1707-1715. https://doi.org/10.1212/WNL.0000000000 004521

7. Besser LM, Mock C, Teylan MA, Hassenstab J, Kukull WA, Crary JF (2019) Differences in cognitive impairment in primary age-related tauopathy versus alzheimer disease. J Neuropathol Exp Neurol. https://doi.org/10. 1093/jnen/nly132 
8. Bishop NA, LuT, Yankner BA (2010) Neural mechanisms of ageing and cognitive decline. Nature 464:529-535. https://doi.org/10.1038/natur e08983

9. Braak H, Braak E (1991) Neuropathological stageing of Alzheimer-related changes. Acta Neuropathol 82:239-259. https://doi.org/10.1007/bf003 08809

10. Braak H, Del Tredici K (2014) Are cases with tau pathology occurring in the absence of Abeta deposits part of the AD-related pathological process? Acta Neuropathol 128:767-772. https://doi.org/10.1007/ s00401-014-1356-1

11. Caillet-Boudin ML, Buee L, Sergeant N, Lefebvre B (2015) Regulation of human MAPT gene expression. Mol Neurodegener 10:28. https://doi.org/ 10.1186/s13024-015-0025-8

12. Cherry JD, Tripodis Y, Alvarez VE, Huber B, Kiernan PT, Daneshvar DH, Mez J, Montenigro PH, Solomon TM, Alosco ML et al (2016) Microglial neuroinflammation contributes to tau accumulation in chronic traumatic encephalopathy. Acta Neuropathol Com 4:UNSP. https://doi.org/10.1186/ s40478-016-0382-8

13. Corrada MM, Sonnen JA, Kim RC, Kawas CH (2016) Microinfarcts are common and strongly related to dementia in the oldest-old: The $90+$ study. Alzheimers Dement 12:900-908. https://doi.org/10.1016/j.jalz.2016.04 006

14. Cosentino S, Scarmeas N, Helzner E, Glymour MM, Brandt J, Albert M, Blacker D, Stern Y (2008) APOE epsilon 4 allele predicts faster cognitive decline in mild Alzheimer disease. Neurology 70:1842-1849. https://doi. org/10.1212/01.wnl.0000304038.37421.cc

15. Crary JF (2016) Primary age-related tauopathy and the amyloid cascade hypothesis: the exception that proves the rule? J Neurol Neuromedicine 1:53-57

16. Crary JF, Trojanowski JQ, Schneider JA, Abisambra JF, Abner EL, Alafuzoff I, Arnold SE, Attems J, Beach TG, Bigio EH et al (2014) Primary age-related tauopathy (PART): a common pathology associated with human aging. Acta Neuropathol 128:755-766. https://doi.org/10.1007/ s00401-014-1349-0

17. Dik MG, Jonker C, Comijs HC, Bouter LM, Twisk JW, van Kamp GJ, Deeg DJ (2001) Memory complaints and APOE-epsilon4 accelerate cognitive decline in cognitively normal elderly. Neurology 57:2217-2222. https:// doi.org/10.1212/wnl.57.12.2217

18. Duyckaerts C, Braak H, Brion JP, Buee L, Del Tredici K, Goedert M, Halliday G, Neumann M, Spillantini MG, Tolnay M et al (2015) PART is part of Alzheimer disease. Acta Neuropathol 129:749-756. https://doi.org/10.1007/ s00401-015-1390-7

19. Farfel JM, Yu L, De Jager PL, Schneider JA, Bennett DA (2016) Association of APOE with tau-tangle pathology with and without beta-amyloid. Neurobiol Aging 37:19-25. https://doi.org/10.1016/j.neurobiolaging.2015.09. 011

20. Farrell $K$, Cosentino S, lida MA, Chapman S, Bennett DA, Faust PL, Louis ED, Crary JF (2019) Quantitative assessment of pathological tau burden in essential tremor: a postmortem study. J Neuropath Exp Neur 78:31-37. https://doi.org/10.1093/jnen/nly104

21. Folstein MF, Robins LN, Helzer JE (1983) The Mini-Mental State Examination. Arch Gen Psychiat 40:812

22. Fox NC, Scahill Rl, Crum WR, Rossor MN (1999) Correlation between rates of brain atrophy and cognitive decline in AD. Neurology 52:1687-1689. https://doi.org/10.1212/Wnl.52.8.1687

23. Giannakopoulos P, Herrmann FR, Bussiere T, Bouras C, Kovari E, Perl DP, Morrison JH, Gold G, Hof PR (2003) Tangle and neuron numbers, but not amyloid load, predict cognitive status in Alzheimer's disease. Neurology 60:1495-1500. https://doi.org/10.1212/01.wnl.0000063311.58879.01

24. Gold G, Bouras C, Kovari E, Canuto A, Glaria BG, Malky A, Hof PR, Michel JP, Giannakopoulos P (2000) Clinical validity of Braak neuropathological staging in the oldest-old. Acta Neuropathol 99:579-582. https://doi.org/ 10.1007/s004010051163 (discussion 583-574)

25. Gomez-Isla T, Hollister R, West H, Mui S, Growdon JH, Petersen RC, Parisi JE, Hyman BT (1997) Neuronal loss correlates with but exceeds neurofibrillary tangles in Alzheimer's disease. Ann Neurol 41:17-24. https://doi. org/10.1002/ana.410410106

26. Hamasaki H, Honda H, Okamoto T, Koyama S, Suzuki SO, Ohara T, Ninomiya T, Kiyohara Y, Iwaki T (2017) Recent increases in hippocampal tau pathology in the aging Japanese population: the hisayama study. J Alzheimers Dis 55:613-624. https://doi.org/10.3233/Jad-160521
27. Hauw JJ, Daniel SE, Dickson D, Horoupian DS, Jellinger K, Lantos PL, McKee A, Tabaton M, Litvan I (1994) Preliminary NINDS neuropathologic criteria for Steele-Richardson-Olszewski syndrome (progressive supranuclear palsy). Neurology 44:2015-2019. https://doi.org/10.1212/wnl.44.11. 2015

28. Hickman RA, Flowers XE, Wisniewski T (2020) Primary age-related tauopathy (PART): addressing the spectrum of neuronal tauopathic changes in the aging brain. Curr Neurol Neurosci Rep 20:39. https://doi.org/10.1007/ s11910-020-01063-1

29. Hyman BT (1998) New neuropathological criteria for Alzheimer disease. Arch Neurol 55:1174-1176. https://doi.org/10.1001/archneur.55.9.1174

30. Jack CR Jr, Bennett DA, Blennow K, Carrillo MC, Dunn B, Haeberlein SB, Holtzman DM, Jagust W, Jessen F, Karlawish J et al (2018) NIA-AA Research Framework: Toward a biological definition of Alzheimer's disease. Alzheimers Dement 14:535-562. https://doi.org/10.1016/j.jalz.2018. 02.018

31. Jack CR, Petersen RC, Xu Y, O'Brien PC, Smith GE, Ivnik RJ, Boeve BF, Tangalos EG, Kokmen E (2000) Rates of hippocampal atrophy correlate with change in clinical status in aging and AD. Neurology 55:484-489. https:// doi.org/10.1212/Wnl.55.4.484

32. Jefferson-George KS, Wolk DA, Lee EB, McMillan CT (2017) Cognitive decline associated with pathological burden in primary age-related tauopathy. Alzheimers Dement 13:1048-1053. https://doi.org/10.1016/j. jalz.2017.01.028

33. Jellinger KA, Alafuzoff I, Attems J, Beach TG, Cairns NJ, Crary JF, Dickson DW, Hof PR, Hyman BT, Jack CR Jr et al (2015) PART, a distinct tauopathy, different from classical sporadic Alzheimer disease. Acta Neuropathol 129:757-762. https://doi.org/10.1007/s00401-015-1407-2

34. Jellinger KA, Attems J (2007) Neurofibrillary tangle-predominant dementia: comparison with classical Alzheimer disease. Acta Neuropathol 113:107-117. https://doi.org/10.1007/s00401-006-0156-7

35. Josephs KA, Murray ME, Tosakulwong N, Whitwell JL, Knopman DS, Machulda MM, Weigand SD, Boeve BF, Kantarci K, Petrucelli L et al (2017) Tau aggregation influences cognition and hippocampal atrophy in the absence of beta-amyloid: a clinico-imaging-pathological study of primary age-related tauopathy (PART). Acta Neuropathol 133:705-715. https:// doi.org/10.1007/s00401-017-1681-2

36. Kovacs GG (2015) Invited review: Neuropathology of tauopathies: principles and practice. Neuropathol Appl Neurobiol 41:3-23. https://doi.org/ 10.1111/nan.12208

37. Kovacs GG, Ferrer I, Grinberg LT, Alafuzoff I, Attems J, Budka H, Cairns NJ, Crary JF, Duyckaerts C, Ghetti B et al (2016) Aging-related tau astrogliopathy (ARTAG): harmonized evaluation strategy. Acta Neuropathol 131:87-102. https://doi.org/10.1007/s00401-015-1509-x

38. Kvitting AS, Fallman K, Wressle E, Marcusson J (2019) Age-Normative MMSE data for older persons aged 85 to 93 in a longitudinal swedish cohort. J Am Geriatr Soc 67:534-538. https://doi.org/10.1111/jgs.15694

39. Markesbery WR (1997) Neuropathological criteria for the diagnosis of Alzheimer's disease. Neurobiol Aging 18:S13-19. https://doi.org/10.1016/ s0197-4580(97)00064-x

40. McKee AC, Cairns NJ, Dickson DW, Folkerth RD, Keene CD, Litvan I, Perl DP, Stein TD, Vonsattel JP, Stewart W et al (2016) The first NINDS/NIBIB consensus meeting to define neuropathological criteria for the diagnosis of chronic traumatic encephalopathy. Acta Neuropathol 131:75-86. https:// doi.org/10.1007/s00401-015-1515-z

41. McKee AC, Stein TD, Crary JF, Bieniek KF, Cantu RC, Kovacs GG (2020) Practical considerations in the diagnosis of mild chronic traumatic encephalopathy and distinction from age-related tau astrogliopathy. J Neuropathol Exp Neurol 79:921-924. https://doi.org/10.1093/jnen/nlaa0 47

42. McKee AC, Stein TD, Nowinski CJ, Stern RA, Daneshvar DH, Alvarez VE, Lee HS, Hall G, Wojtowicz SM, Baugh CM et al (2013) The spectrum of disease in chronic traumatic encephalopathy. Brain 136:43-64. https://doi.org/10. 1093/brain/aws307

43. Mirra SS, Heyman A, McKeel D, Sumi SM, Crain BJ, Brownlee LM, Vogel FS, Hughes JP, van Belle G, Berg L (1991) The consortium to establish a registry for Alzheimer's Disease (CERAD). Part II. standardization of the neuropathologic assessment of alzheimer's disease. Neurology 41:479-486

44. Mock C, Teylan M, Beecham G, Besser L, Cairns NJ, Crary JF, Katsumata Y, Nelson PT, Kukull W (2020) The Utility of the National Alzheimer's coordinating center's database for the rapid assessment of evolving 
neuropathologic Conditions. Alzheimer Dis Assoc Disord 34:105-111. https://doi.org/10.1097/WAD.0000000000000380

45. Montine TJ, Phelps CH, Beach TG, Bigio EH, Cairns NJ, Dickson DW, Duyckaerts C, Frosch MP, Masliah E, Mirra SS et al (2012) National Institute on Aging-Alzheimer's Association guidelines for the neuropathologic assessment of Alzheimer's disease: a practical approach. Acta Neuropathol 123:1-11. https://doi.org/10.1007/s00401-011-0910-3

46. Morris JC (1993) The Clinical Dementia Rating (CDR): current version and scoring rules. Neurology 43:2412-2414

47. Morris M, Maeda S, Vossel K, Mucke L (2011) The many faces of tau. Neuron 70:410-426. https://doi.org/10.1016/j.neuron.2011.04.009

48. Nelson PT, Dickson DW, Trojanowski JQ, Jack CR, Boyle PA, Arfanakis K, Rademakers R, Alafuzoff I, Attems J, Brayne C et al (2019) Limbic-predominant age-related TDP-43 encephalopathy (LATE): consensus working group report. Brain 142:1503-1527. https://doi.org/10.1093/brain/awz099

49. Pierce AL, Kawas CH (2017) Dementia in the oldest old: Beyond Alzheimer disease. PLoS Med 14:e1002263. https://doi.org/10.1371/journal. pmed. 1002263

50. Planche V, Coupe P, Helmer C, Le Goff M, Amieva H, Tison F, Dartigues JF, Catheline G (2019) Evolution of brain atrophy subtypes during aging predicts long-term cognitive decline and future Alzheimer's clinical syndrome. Neurobiol Aging 79:22-29. https://doi.org/10.1016/j.neurobiola ging.2019.03.006

51. Quintas-Neves M, Teylan MA, Besser L, Soares-Fernandes J, Mock CN, Kukull WA, Crary JF, Oliveira TG (2019) Magnetic resonance imaging brain atrophy assessment in primary age-related tauopathy (PART). Acta Neuropathol Commun 7:204. https://doi.org/10.1186/s40478-019-0842-z

52. Robinson AC, Davidson YS, Roncaroli F, Minshull J, Tinkler P, Horan MA, Payton A, Pendleton N, Mann DMA (2021) Early changes in visuospatial episodic memory can help distinguish primary age-related tauopathy from Alzheimer's disease. Neuropathol Appl Neurobiol. https://doi.org/ 10.1111/nan.12726

53. Robinson JL, Corrada MM, Kovacs GG, Dominique M, Caswell C, Xie SX, Lee VMY, Kawas CH, Trojanowski JQ (2018) Non-Alzheimer's contributions to dementia and cognitive resilience in The 90+Study. Acta Neuropathol 136:377-388. https://doi.org/10.1007/s00401-018-1872-5

54. Serrano-Pozo A, Qian J, Monsell SE, Betensky RA, Hyman BT (2015) APOE epsilon 2 is associated with milder clinical and pathological Alzheimer disease. Ann Neurol 77:917-929. https://doi.org/10.1002/ana.24369

55. Shi Y, Yamada K, Liddelow SA, Smith ST, Zhao L, Luo W, Tsai RM, Spina S, Grinberg LT, Rojas JC et al (2017) ApoE4 markedly exacerbates taumediated neurodegeneration in a mouse model of tauopathy. Nature 549:523-527. https://doi.org/10.1038/nature24016
56. Signaevsky M, Prastawa M, Farrell K, Tabish N, Baldwin E, Han N, lida MA, Koll J, Bryce C, Purohit D et al (2019) Artificial intelligence in neuropathology: deep learning-based assessment of tauopathy. Lab Invest 99:1019-1029. https://doi.org/10.1038/s41374-019-0202-4

57. Small BJ, Graves AB, McEvoy CL, Crawford FC, Mullan M, Mortimer JA (2000) Is APOE-epsilon 4 a risk factor for cognitive impairment in normal aging? Neurology 54:2082-2088. https://doi.org/10.1212/Wnl.54.11.2082

58. Small BJ, Rosnick CB, Fratiglioni L, Backman L (2004) Apolipoprotein E and cognitive performance: a meta-analysis. Psychol Aging 19:592-600. https://doi.org/10.1037/0882-7974.19.4.592

59. Smith VD, Bachstetter AD, Ighodaro E, Roberts K, Abner EL, Fardo DW, Nelson PT (2018) Overlapping but distinct TDP-43 and tau pathologic patterns in aged hippocampi. Brain Pathol 28:264-273. https://doi.org/ 10.1111/bpa. 12505

60. Teylan M, Besser LM, Crary JF, Mock C, Gauthreaux K, Thomas NM, Chen YC, Kukull WA (2019) Clinical diagnoses among individuals with primary age-related tauopathy versus Alzheimer's neuropathology. Lab Invest 99:1049-1055. https://doi.org/10.1038/s41374-019-0186-0

61. Teylan M, Mock C, Gauthreaux K, Chen YC, Chan KCG, Hassenstab J, Besser LM, Kukull WA, Crary JF (2020) Cognitive trajectory in mild cognitive impairment due to primary age-related tauopathy. Brain 143:611-621. https://doi.org/10.1093/brain/awz403

62. Walker JM, Richardson TE, Farrell K, lida MA, Foong C, Shang P, Attems J, Ayalon G, Beach TG, Bigio EH et al (2021) Early selective vulnerability of the CA2 hippocampal subfield in primary age-related tauopathy. J Neuropathol Exp Neurol 80:102-111. https://doi.org/10.1093/jnen/nlaa1 53

63. West MJ, Slomianka L, Gundersen HJ (1991) Unbiased stereological estimation of the total number of neurons in thesubdivisions of the rat hippocampus using the optical fractionator. Anat Rec 231:482-497. https://doi.org/10.1002/ar.1092310411

64. Zhang X, Sun B, Wang X, Lu H, Shao F, Rozemuller AJM, Liang H, Liu C, Chen J, Huang M et al (2019) Phosphorylated TDP-43 staging of primary age-related tauopathy. Neurosci Bull 35:183-192. https://doi.org/10. 1007/s12264-018-0300-0

65. Zhukareva V, Trojanowski JQ, Lee VM (2004) Assessment of pathological tau proteins in frontotemporal dementias: qualitative and quantitative approaches. Am J Geriatr Psych 12:136-145

\section{Publisher's Note}

Springer Nature remains neutral with regard to jurisdictional claims in published maps and institutional affiliations.
Ready to submit your research? Choose BMC and benefit from:

- fast, convenient online submission

- thorough peer review by experienced researchers in your field

- rapid publication on acceptance

- support for research data, including large and complex data types

- gold Open Access which fosters wider collaboration and increased citations

- maximum visibility for your research: over 100M website views per year

At BMC, research is always in progress.

Learn more biomedcentral.com/submissions 